\title{
Docentes universitarios. Una formación centrada en la práctica
}

\author{
Pilar Azcárate \\ Universidad de Cádiz. Área Didáctica de la Matemática. Departamento de Didáctica. \\ Facultad de Ciencias de la Educación. Cádiz España. pilar.azcarate@uca.es \\ ORCID:https://orcid.org/0000-0002-1306-5140
}

\section{University teachers. Practice-focused training}

\begin{abstract}
Para citar la obra reseñada: Azcárate, P (2020). Reseña de Alba-Fernández, N. y Porlán, R. (Coordinadores) (2020). Docentes universitarios. Una formación centrada en la práctica. Madrid: EDICIONES MORATA, S. L, 336 págs. ISBN: 9788471129789. ISBN ebook: 978-84-7112-979-6. Revista de Educación Ambiental y Sostenibilidad $2(1), \quad 1402 . \quad$ doi: 10.25267/Rev_educ_ambient_sostenibilidad.2020.v2.i1.1402
\end{abstract}

La educación del profesorado universitario pocas veces es objeto de una profunda revisión y de propuestas valiosas para la mejora de su práctica docente. En este libro se abordan aspectos relevantes relacionados con la formación del profesorado universitario, centrados, en una primera parte, en la presentación y reflexión sobre diferentes estrategias formativas que giran en torno a la estrategia CIMA (Ciclos de Mejora en el Aula) y en una segunda parte, con la presentación de casos concretos de procesos de formación del Programa FIDOP (Formación e Innovación Docente del Profesorado) y sus implicaciones.

En la primera parte del libro se presenta, una revisión interesante sobre investigaciones relativas a la formación de docentes universitarios y, diversas perspectivas formativas apoyadas en diferentes estrategias. En cada caso, presentan la estrategia y aportan indicaciones útiles para la formación del docente y su posible incidencia en el aula universitaria.

Comienzan con aquellas que ponen el foco en la actividad del alumnado y su implicación directa en el proceso. Apuestan por introducir en el aula universitaria metodologías activas basadas en la indagación y promoviendo logros de aprendizaje útil y duradero.

Otra estrategia interesante es la que se organizan en torno a la Lesson Study que apoya la indagación en el aula como la columna vertebral de los procesos formativos. Utiliza la Investigación desde una perspectiva cooperativa en su interacción de aula. Los grupos de docentes universitarios diseñan, desarrollan, observan, analizan y revisan una propuesta (Lesoon), estudiada desde la perspectiva de su incidencia en el aprendiz, vinculando su práctica a la investigación, buscando su mejora.

La estrategia conocida como SoTL enfatiza el papel de las evidencias de aprendizaje del alumnado, las cuales promueven controversias y originan la necesidad de explorar sobre su significado. Estrategia que relaciona los procesos de enseñanza y aprendizaje de una disciplina, con el estudio y la investigación dentro de la propia disciplina, su estructura y su forma de pensar. Trabajar desde el núcleo de la disciplina permite abordar los procesos formativos como un problema académico relevante dentro de la propia disciplina. 
En el último capítulo de esta primera parte se presenta y analiza el programa formativo FIDOP, desarrollado en la Universidad de Sevilla dirigido a la formación de sus docentes. Este programa se apoya en los llamados Ciclos de Mejora en el Aula (CIMA). Esta estrategia es una integración equilibrada de las presentadas en los capitulo previos. Ligando los procesos de mejora de la practica docentes universitaria a la investigación en la propia aula universitaria. Proponen y desarrollan los 10 pasos del Ciclo de Mejora, analizando su sentido, las diferentes actividades y cuestiones que se pueden ir planteando.

Los ciclos de mejora es una estrategia formativa centrada en la práctica del profesorado universitario. Es una formación orientada, centrada en la práctica, dirigida a la toma de conciencia de su propia realidad por el docente, que se apoya en su investigación y observación continua del aula. Es una formación gradual, colegiada y sostenida en el tiempo. Su peculiaridad más significativa, que aporta un valor indiscutible, es una combinación equilibrada ente el aprendizaje entre iguales, promoviendo la relación de grupos de docentes, con el acompañamiento de un experto que permite introducir cuestiones o retos.

En la segunda parte del libro se presenta una investigación sobre los resultados del propio Programa FIDOP. Presentan las experiencias desarrolladas con diferentes grupos de docentes universitarios de diversos campos de conocimientos que han participado en FIDOP y sus respectivos Ciclos de Mejora. En ellos se refleja una exhaustiva descripción de las fases seguidas en el proceso formativo, las actividades propuestas y los instrumentos de recogida de información utilizados para la investigación, así como, el sistema de categoría utilizados para el análisis de las producciones de los docentes. Su objetivo es presentar, los resultados que, en términos de cambio docente, se producen en las concepciones y en la práctica de un grupo de participantes en el programa.

Así, el sexto capítulo, se dedica a la descripción de las 6 fases del proceso y de los participantes e instrumentos de la investigación. El siguiente, presenta los resultados del primer estudio, aportando información sobre la evolución de los modelos docentes universitarios. En los dos últimos capítulos del libro, de los nueves casos que configuran el estudio, se describen los resultados concretos de dos casos, uno de Ciencia y Tecnología (Ingeniería) y otro de Ciencias Sociales (Educación). En los cuales se presentan las progresiones y obstáculos que se han detectado en su proceso hacia la mejora docente.

En definitiva, este libro establece este diálogo tan necesario entre la práctica docente universitaria y sus fundamentos didácticos. Este texto representa una herramienta extraordinaria para ayudar a conectar investigación y práctica docente universitaria. Aporta claves eficaces para la puesta en marcha de programas de formación de docentes universitarios, y formula una propuesta de hipótesis de progresión del cambio docente en la universidad. De hecho, se trata de un conjunto de ideas y orientaciones enormemente útiles y prácticas, con un objetivo muy claro, mejorar la docencia universitaria. 